\title{
sciendo
}

\section{PATERNAL LEAKAGE OF MITOCHONDRIAL DNA IN THE RACCOON DOG (NYCTEREUTES PROCYONOIDES GRAY 1834)}

\author{
Sylwia Nisztuk-Pacek ${ }^{1}$, Brygida Ślaska ${ }^{1 \star}$, Ludmiła Grzybowska-Szatkowska², Marek Babicz ${ }^{3}$ \\ ${ }^{1}$ Institute of Biological Bases of Animal Production, Faculty of Biology, Animal Sciences \\ and Bioeconomy, University of Life Sciences in Lublin, Akademicka 13, 20-950 Lublin, Poland \\ ${ }^{2}$ Department of Oncology, Medical University of Lublin, Jaczewskiego 7, 20-090 Lublin, Poland \\ ${ }^{3}$ Institute of Animal Breeding and Biodiversity Conservation, Faculty of Biology, Animal Sciences \\ and Bioeconomy, University of Life Sciences in Lublin, Akademicka 13, 20-950 Lublin, Poland \\ •Corresponding author: brygida.slaska@up.lublin.pl
}

\begin{abstract}
The aim of the study was to describe the mechanism of mitochondrial DNA inheritance in a group of farmed raccoon dogs. The study involved 354 individuals. Whole peripheral blood was the research material. DNA was isolated and PCR was performed for two fragments of mitochondrial genes: COX1 (cytochrome oxidase subunit 1 gene) and COX2 (cytochrome oxidase subunit 2 gene). The PCR products were sequenced and subjected to bioinformatics analyses. Three mitochondrial haplotypes were identified in the $C O X 1$ gene fragment and two in the $C O X 2$ gene fragment. The analysis of mtDNA inheritance in the paternal line confirmed the three cases of paternal mtDNA inheritance, i.e. the so-called "paternal leakage" in the analysed population. In two families, all offspring inherited paternal mitochondrial DNA, whereas in one family one descendant inherited paternal mtDNA and another one inherited maternal mtDNA. The lineage data indicated that one female which inherited maternal mitochondrial DNA transferred it onto the next generation. To sum up, the results of the study for the first time demonstrated the phenomenon of "paternal leakage" in farmed raccoon dogs, which facilitated description of mitochondrial DNA inheritance in the paternal line.
\end{abstract}

Key words: $C O X 1$ gene, $C O X 2$ gene, paternal inheritance, polymorphism, raccoon dog

The mitochondrial genome (mtDNA) is present in the cell cytoplasm in a great number of copies, which depends on the energy requirements of tissues in the organism. The animal mitochondrial genome comprises in total 37 genes, i.e. 2 ribosomal RNA genes, 22 transfer RNA genes, and 13 genes encoding respiratory chain proteins. The only non-coding sequence in the mitochondrial DNA is the D-loop, which is characterised by the highest degree of polymorphism. The primary function of proteins encoded by mtDNA genes is their involvement in the cellular respiration process with its basic function involving release and storage of energy in 
high-energy bonds in ATP (Higuchi, 2007; Grzybowska-Szatkowska and Slaska, 2012).

A characteristic feature of the mitochondrial genome is the absence of recombination. Changes in mtDNA appearing over time are a result of cellular adaptation to the changing external factors and are designed to adapt the organism to new conditions. Simultaneously, the mitochondrial genome is usually inherited in the maternal line. These characteristic traits facilitate the use of mtDNA in various investigations concerning evolution, individual identification, forensic genetics, diagnostics, and the process of aging and apoptosis (Zhao et al., 2004; Slaska and Grzybowska-Szatkowska, 2011; Grzybowska-Szatkowska and Slaska, 2012).

It has been assumed for many years that mitochondrial DNA is inherited only in the maternal line. During fertilisation, spermatid mitochondria penetrate into the oocyte, but their number is negligible in comparison with the number of oocyte mitochondria. Additionally, in early embryogenesis, spermatid mitochondria disappear through selective destruction or inactivation or due to the substantial excess of oocyte mitochondria (Schwartz and Vissing, 2002). However, the research conducted for the last several years has demonstrated that sometimes inheritance of mitochondrial DNA can occur in the paternal line, e.g. in bees (Meusel and Moritz, 1993), humans (Schwartz and Vissing, 2002), mice (Gyllensten et al., 1991), poultry (Alexander et al., 2015), cattle (Steinborn et al., 1998), or sheep (Zhao et al., 2004). Simultaneously, natural uniparental inheritance was found in mussels (Mytilus edulis), in which males and females transferred their mitochondrial DNA onto male descendants and all offspring, respectively (Sutherland et al., 1998). The available literature does not provide information about the so-called "paternal leakage" in fur-bearing animals from the family Canidae. Identification of inheritance of mitochondrial DNA in the paternal line in populations of farmed animals is highly important, as this phenomenon can be regarded as a genetic factor exerting a significant effect on evolution, development of diseases, or the value of production traits in these animals. In evolution studies, this phenomenon can complicate the reconstruction of phylogenetic trees. To date, the construction of dendrograms has been based on an assumption that mtDNA is inherited in the female line (Fontaine et al., 2007). In the case of livestock, it is essential to determine whether maternal mtDNA has been replaced completely by paternal mtDNA or whether heteroplasmy has occurred. The ratio of both types of mitochondrial DNA in the cell may be reflected in the physiological effects of this phenomenon, including the values of performance traits.

Therefore, the aim of the study was to analyse mitochondrial DNA inheritance in a group of farmed raccoon dogs.

\section{Material and methods}

The investigations were conducted in a group of 354 raccoon dogs maintained in a fur-bearing animal farm located in south-eastern Poland. The analysed lineage information of a 4-generation population of raccoon dogs was taken from the documentation available on the breeding farm. 
Whole peripheral blood taken intravitally into sterile vacuum tubes containing the $\mathrm{K}_{2}$ EDTA anticoagulant was used in the genetic analyses. The biological material was used for isolation of total DNA, which was performed with the commercial QIAGEN DNeasy Blood \& Tissue Kit according to manufacturer's procedure. The quality and quantity of the isolated genomic DNA were determined using agarose gel electrophoresis and a spectrophotometric method, respectively.

The lineage data obtained from the breeding documentation were screened by analysis of 20 microsatellite sequences (according to Ślaska, 2010). The results were used to verify the lineages. All inaccuracies were corrected by comparison with the results of the molecular analyses. Thus, errors that had arisen at the stage of compilation of the documentation on the farm were eliminated.

The isolated genetic material was used to amplify the selected fragments of mitochondrial genes $(C O X 1, C O X 2)$ using the PCR technique. The reaction mixture was prepared in an AURA Vertical laminar chamber. PCR was performed in a Labcycler (SensoQuest) thermocycler. The primer sequences for the fragment of the mitochondrial COX1 gene (m.5616-6272 - F: CCGGACATGGCATTCCCCCG, R: GGCGGACGTAAAGTACGCTCGTG) were designed using the Primer-BLAST program (http://www.ncbi.nlm.nih.gov/tools/primer-blast/). Primers specified in the literature were used for the fragment of the COX2 genes (m.7048-7713 - F: TACCCTTTCCAACTCGGATT, R: GGCAGATCAGGTTTCGAAAT) (Slaska and GrzybowskaSzatkowska, 2011).

The temporal temperature profile of the $\mathrm{PCR}$ reaction consisted of initial denaturation $\left(95^{\circ} \mathrm{C}, 10 \mathrm{~min}\right), 35$ denaturation cycles $\left(95^{\circ} \mathrm{C}, 1 \mathrm{~min}\right)$, primer annealing $\left(52^{\circ} \mathrm{C}\right.$ - COX1, $\left.57^{\circ} \mathrm{C}-C O X 2, C Y T B, 1 \mathrm{~min}\right)$ and extension $\left(72^{\circ} \mathrm{C}, 1 \mathrm{~min}\right)$, and final primer extension $\left(72^{\circ} \mathrm{C}, 20 \mathrm{~min}\right)$. The gene fragment amplicons were sequenced using a sequencing BigDye Terminator v1.1 Cycle Sequencing Kit (Applied Biosystems, Foster City, CA, USA). Purified products were sequenced on an ABI 377 automated sequencer (Applied Biosystems, Foster City, CA, USA). The nucleotide sequences obtained were subjected to bioinformatics analysis carried out with the use of the DNA Baser Sequence Assembler v4 (2013).

The encoding sequences of the raccoon dog COX1 (cytochrome c oxidase subunit I) and $C O X 2$ (cytochrome c oxidase subunit II) genes were deposited in the GenBank under accession numbers KX467623 and KX467624, respectively. According to our own results, analysis of the $C O X 1$ gene sequence revealed the presence of seven polymorphisms, which were grouped into three mitochondrial haplotypes: A (TTCCTTC - in positions m.5715, m.5772, m.5931, m.6063, m.6090, m.6096, and m.6159, respectively), B (TCCCTTC), and C (CTTTCCT). The analysis of the $C O X 2$ gene sequence identified six polymorphisms in the group of the raccoon dogs, grouped into two haplotypes: A (AAGAAC - in positions m.7125, m.7194, m.7221, m.7287, m.7578, and m.7665, respectively) and B (GCAGGT). In this way, five mitochondrial haplogroups were identified (Nisztuk-Pacek, 2016). The study was approved by the II Local Ethical Commission for animal experiments in Lublin, Poland (resolution number 9/2008). 


\section{Results}

The analysis of mitochondrial DNA inheritance performed for fourteen families of raccoon dogs showed inheritance of mtDNA in the paternal line, different from the classic inheritance, in three families. The occurrence of "paternal leakage" was supported by the analysis of inheritance of the identified mitochondrial haplotypes and haplogroups between related individuals (Figure 1). Paternal mtDNA inheritance in all offspring was detected in two cases. In one case, one descendant inherited paternal mitochondrial DNA and another individual inherited maternal mtDNA (Table 1). Additionally, the lineage data revealed that female K46 (family I), which inherited paternal mitochondrial DNA, transferred it onto the next generation (Figure 2).
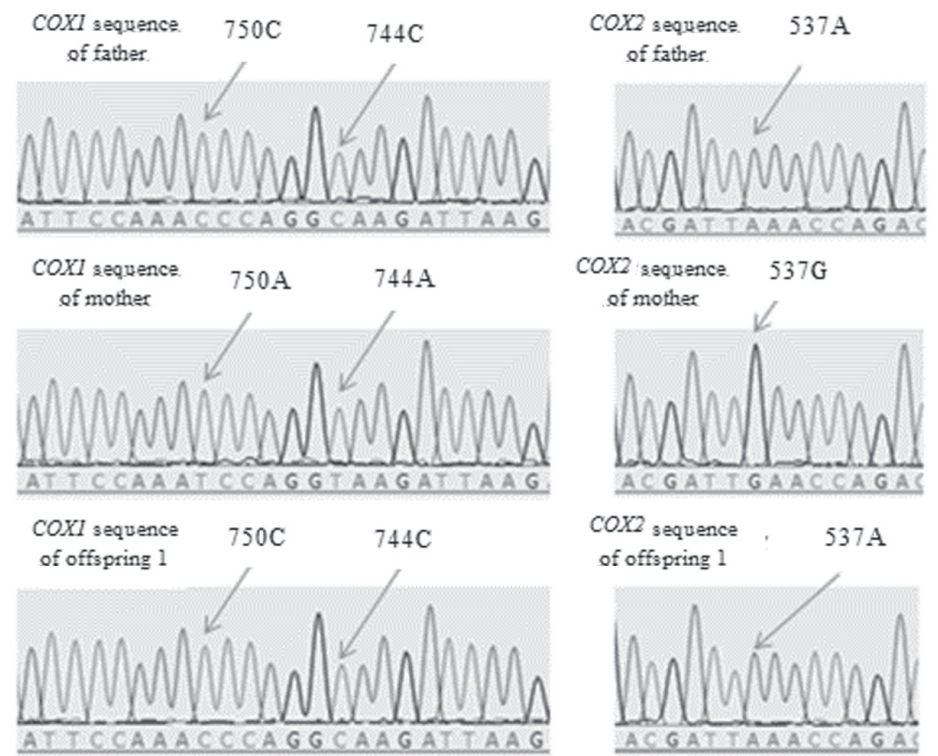

of offspring 1
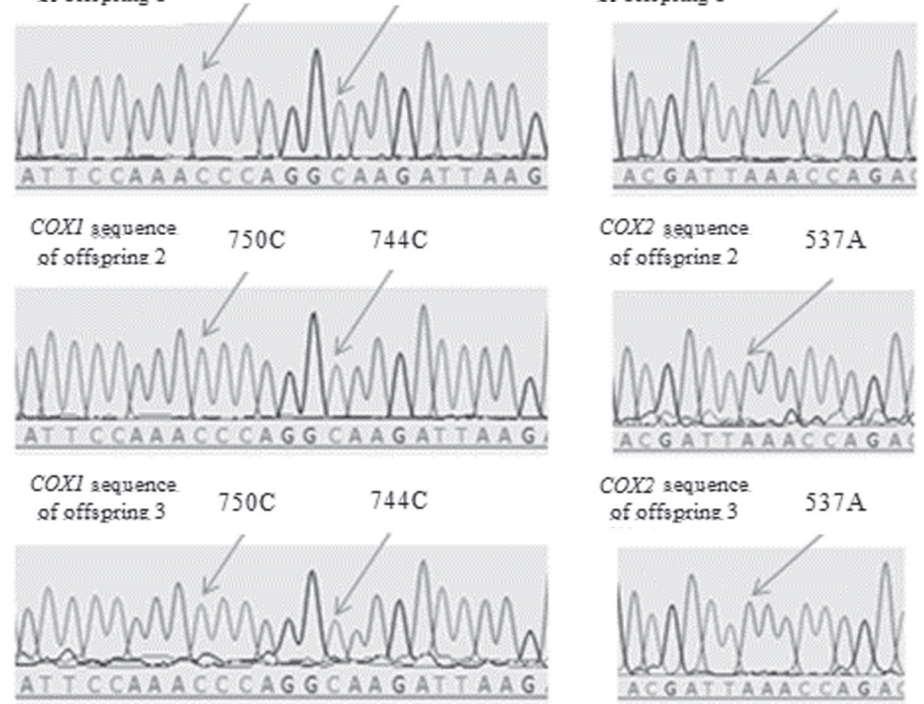

Figure 1. Electropherograms of $C O X 1$ and $C O X 2$ gene fragments presenting examples of mtDNA inheritance in the paternal line in the population of the farmed raccoon dogs (family II) 
Table 1. Identified cases of the so-called "paternal leakage" in the group of the farmed raccoon dogs

\begin{tabular}{|c|c|c|c|}
\hline & $\begin{array}{c}\text { Individual - number in the breed- } \\
\text { ing documentation }\end{array}$ & Haplotype $C O X 1$ & Haplotype $C O X 2$ \\
\hline \multirow{4}{*}{ Family I } & Father $-\mathrm{H} 3$ & A (TTCCTTC) & B (GCAGGT) \\
\hline & Mother - H18 & C (CTTTCCT) & A (AAGAAC) \\
\hline & Offspring $1(\widehat{\jmath})-\mathrm{K} 51$ & A (TTCCTTC) & B (GCAGGT) \\
\hline & Offspring $2(q)-\mathrm{K} 46$ & A (TTCCTTC) & B (GCAGGT) \\
\hline \multirow{5}{*}{ Family II } & Father - H39 & C (CTTTCCT) & A (AAGAAC) \\
\hline & Mother - H2 & A (TTCCTTC) & B (GCAGGT) \\
\hline & Offspring $1(ふ)-L 45$ & C (CTTTCCT) & A (AAGAAC) \\
\hline & Offspring $2(+)-$ L56 & C (CTTTCCT) & A (AAGAAC) \\
\hline & Offspring $3(+)$ - L58 & C (CTTTCCT) & A (AAGAAC) \\
\hline \multirow{4}{*}{ Family III } & Father - L5 & C (CTTTCCT) & A (AAGAAC) \\
\hline & Mother - L80 & A (TTCCTTC) & A (AAGAAC) \\
\hline & Offspring $1(+)$ - N94 & A (TTCCTTC) & A (AAGAAC) \\
\hline & Offspring $2(+)-\mathrm{N} 96$ & C (CTTTCCT) & A (AAGAAC) \\
\hline
\end{tabular}

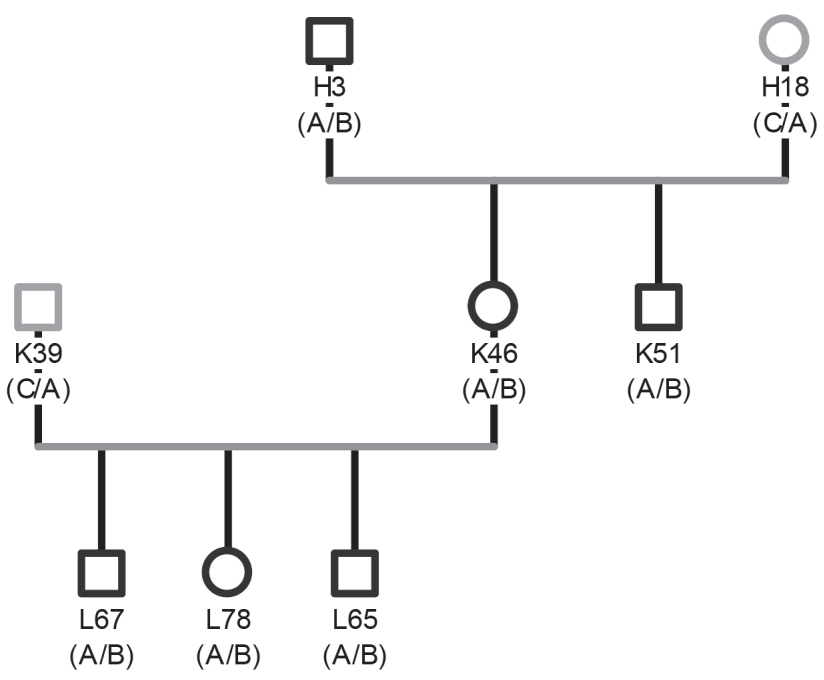

Figure 2. Phylogenetic tree showing mtDNA inheritance in family I with the subsequent generation number of the animal (haplotype)

\section{Discussion}

Reports of mitochondrial DNA inheritance in the paternal line in different animal species arouse great interest among researchers. This is related to the existence of increasing scientifically proven evidence for this phenomenon. To date, two possible 
mechanisms explaining this type of mtDNA inheritance have been described. One of them is the penetration of spermatid mitochondria into the egg cell at the time of fertilisation (Cummins et al., 1998). Paternal mitochondrial DNA is then present in embryos at a low level, compared with the oocyte mtDNA. The other mechanism is the phenomenon of mitochondrial genome recombination in the mitochondrion. The recombination can occur between paternal and maternal mitochondrial genomes (Zhao et al., 2004).

The present study has confirmed the phenomenon of inheritance of mitochondrial DNA in the paternal line in the group of the farmed raccoon dogs, which has not been reported in the literature until now. "Paternal leakage" has been evidenced by the analysis of haplotypes in the verified animal lineages. Furthermore, noteworthy is the fact that no heteroplasmy was found in any of the analysed animals or any of the mtDNA fragments. This indicates complete replacement of maternal mtDNA by paternal mtDNA. In one family, paternal mtDNA was detected in one descendant, while the other individual inherited maternal mitochondrial DNA. It can therefore be assumed that both maternal and paternal mtDNA is present in an early stage of embryonic development in all raccoon dogs, but only one form is able to persist throughout the later stages of development. The persistence of one type of mtDNA can be explained by the theory of how mtDNA reaches homoplasmy. There are two theories elucidating the cell homoplasmy phenomenon. In the presence of two mtDNA types in the cell, intramitochondrial selection takes place and one type of mtDNA dominates (functional advantage) in the mitochondrion. During division, the cell is targeted at establishing the dominance of one type of mtDNA, i.e. homoplasmy (replicative segregation) (Gattermann et al., 2002; Pejovic et al., 2004). The division is accompanied by segregation of mitochondria in the cell cytoplasm and daughter cells receive only one type of mtDNA. With the successive cell divisions, only one cell type can dominate and homoplasmy can take place. It can also occur through random segregation of mitochondria during cell divisions (mathematical model of homoplasmy). In subsequent generations of daughter cells, heteroplasmy may persist or homoplasmy can take place due to genetic drift (Lyamzaev et al., 2004; Gattermann et al., 2002). Genetic drift leads to elimination or fixation of rare mtDNA variants. Synonymous polymorphisms can probably be associated with hardly identifiable mutations leading to replacement of wild-type mtDNA and, in the case analysed in this study, replacement of maternal mtDNA by paternal mtDNA (Ishii et al., 2006). Most probably, mitochondria control their replication. It is a puzzling question, however, how sense-type polymorphisms could modulate the process of translation (Ishii et al., 2006; Brandon et al., 2006). Possibly, in the case of the analysed group of farmed animals, there were external factors promoting paternal mtDNA during embryogenesis. Environmental adaptation may have taken place at this stage. Changes occurring in human mtDNA and formation of specific human mitochondrial groups and haplogroups were associated with migration of man across continents and with climate change. These changes have an adaptive nature. In this case, sense and missense polymorphisms take place. The selective growth of cells with one type of mtDNA may also be related to the nDNA genotype, which stimulates division in cells with specific mtDNA, resulting in a homogeneous population 
of cells. It cannot be excluded that it results from interactions between mtDNA and nDNA. The individual raccoon dogs inherited a different nuclear genotype and different phenotypic traits, which resulted in different energy demand of the cells and required promotion of a different type of mtDNA.

The first scientific reports of "paternal leakage" presented the phenomenon in fruit flies, mice, and honeybees (Kondo et al., 1990; Gyllensen et al., 1991; Meusel and Moritz, 1993). Kondo et al. (1990) demonstrated the "paternal leakage" phenomenon in four lines of the fruit fly (Drosophila). In three lines, maternal mtDNA was completely replaced by paternal mtDNA, whereas flies from the fourth family were characterised by heteroplasmy (both maternal and paternal mtDNA). Similarly, the presence of paternal mtDNA was detected in mouse progeny of two inbred strains, whose mitochondrial genomes were easy to distinguish. In this case, paternal mtDNA detected in the offspring exhibited a level of $10^{-4}$ relative to maternal $\mathrm{mtD}$ NA (Gyllensen et al., 1991). An interesting example is the honeybee (Apis mellifera). Fertilisation in this species is characterised by polyspermy. The entire sperm enters the oocyte, which significantly enhances the probability of inheritance of paternal mitochondrial DNA. Analyses carried out in two subspecies of honeybees revealed the presence of $27 \%$ of paternal mitochondrial DNA in the fertilised oocyte relative to the total mtDNA. This amount slowly declined during the subsequent stages of development. Either no paternal mtDNA or trace amounts thereof were detected in adult individuals. The results of these analyses support the theory of the presence of spermatid mitochondrial DNA in the early embryogenesis stage in different species (Meusel and Moritz, 1993).

A spectacular discovery was the detection of "paternal leakage" in humans as well (Schwartz and Vissing, 2002). A 28-year-old mitochondrial myopathy patient was analysed. It was found in the analysis of mtDNA haplotypes that the mitochondrial genome had a paternal origin and accounted for $90 \%$ of the mitochondrial DNA located in patient's muscles. This discovery supports the rationale behind research on "paternal leakage", particularly in the case of sporadic mutations that are difficult to explain by science (Schwartz and Vissing, 2002).

The "paternal leakage" phenomenon was also investigated in livestock, including sheep and poultry (Zhao et al., 2004; Alexander et al., 2015). Sheep lineages were screened with 12 highly polymorphic microsatellite markers to confirm relatedness (Zhao et al., 2004). Cases of "paternal leakage" were observed in two families of sheep, which had the same mother and different fathers. PCR-RFLP analysis demonstrated paternal mtDNA inheritance (Zhao et al., 2004). Similarly, mtDNA inheritance in the paternal line was evidenced in a poultry population (Alexander et al., 2015). Analysis of the inheritance of mutations in genes ND4L and CYTBu by pedigree individuals proved inheritance of paternal mitochondrial DNA in one line of the poultry. The results obtained by the authors and the present results suggest that the "paternal leakage" phenomenon is not as rare as is commonly assumed. It should be kept in mind that the leakage is at present impossible to detect when maternal and paternal mtDNA represent the same haplotype. This suggests, therefore, existence of possible cases of inheritance of mtDNA in the paternal line that are undetectable with the available molecular methods (Fontaine et al., 2007). 
In summary, the results obtained in the study are the first literature data on the so-called "paternal leakage" in farmed raccoon dogs and they contribute to the description of inheritance of mitochondrial DNA in the paternal line. Simultaneously, the results of the analysis suggest that parental mtDNA inheritance can take place in different animal species more often than indicated by the scarce information provided by available literature.

\section{References}

Alexander M., Ho S.Y.W., Molak M., Barnett R., Carloborg Ö., Dorshorst B., Honaker C., Aw a dalla P., B esnier F., Wahlberg P., Dobney K., Si e gel P., Andersson L., Larson G. (2015). Mitogenomic analysis of a 50-generation chicken pedigree reveals a rapid rate of mitochondrial evolution and evidence for paternal mtDNA inheritance. Biol. Letters, 11: 20150561.

B rand on M., B a ldi P., W a ll a ch D.C. (2006). Mitochondrial mutations in cancer. Oncogene, 25: $4647-4662$.

Cummins J.M., Wakay a ma T., Yanag imachi R. (1998). Fate of microinjected spermatid mitochondria in the mouse oocyte and embryo. Zygote, 6: 213-222.

F o n t a i n e K.M., C o o l e y J.R., S i m on C. (2007). Evidence for paternal leakage in hybrid periodical cicadas (Hemiptera: Magicicada spp.). PLoS ONE 2(9): e892.

Gattermann N., Wulfert M., Hofh a u G. (2002). How frequent is mutation in the mitochondrial cytochrome c oxidase gene in patients with myelodysplastic syndromes? Br. J. Haematol., 119: $1139-1140$.

Grzybowska-Szatkowska L., S laska B. (2012). Mitochondrial DNA and carcinogenesis (review). Mol. Med. Rep., 6: 923-930.

Gyllensten U., Wharton D., Jose fs son A., Wils on A.C. (1991). Paternal inheritance of mitochondrial DNA in mice. Nature, 352: 255-257.

H i g u c hi M. (2007). Regulation of mitochondrial DNA content and cancer. Mitochondrion, 7: 53-57.

I s h i i N., I s h i i T., H a r t m a n P.S. (2006). The role of the electron transport gene SDHC on lifespan and cancer. Exp. Gerontol., 41: 952-656.

Kondo R., Satta Y., Matsuura E.T., Ishiwa H., Takahata N., Chiqusa S.I. (1990). Incomplete maternal transmission of mitochondrial DNA in Drosophila. Genetics, 126: 657-663.

Lyamzaev K.G., Izyumov D.S., Avetisyan A.V., Yang F., Pletjushkina O.Y., Chernyak B.V. (2004). Inhibition of mitochondrial bioenergetics: the effects on structure of mitochondria in the cell and on apoptosis. Acta Biochim. Pol., 51: 553-562.

M e u s e 1 M.S., M o rit z R.F. (1993). Transfer of paternal mitochondrial DNA during fertilization of honeybee (Apis mellifera L.) eggs. Curr. Genet., 24: 539-543.

$\mathrm{N}$ i s z t u k - P a c e k S. (2016). Genetic variability of farmed fur animals of the Canidae family assessed by nuclear and mitochondrial DNA analysis. Med. Weter., 72: 505-510.

Pejovic T., Ladner D., Intengan M., Zheng K., F a irchild T., Dillon D., Eas ley S., Dillon D., Marchetti D., Schwartz P., Lele S., Costa J., Odunsid K. (2004). Somatic D-loop mitochondrial DNA mutations are frequent in uterine serous carcinoma. Eur. J. Cancer, 40: 2519-2524.

S c h w art z M., Vi s s ing J. (2002). Paternal inheritance of mitochondrial DNA. New Engl. J. Med., 347: $576-580$

S laska B., Grzybowska-S zatkowsk a L. (2011). Analysis of the mitochondrial haplogroups of farm and wild-living raccoon dogs in Poland. Mitochondr. DNA, 22: 105-110.

Ste inborn R., Zakhartchenko V., Jelyazkov J., Klein D., Wolf E., Müller M., B r e m G. (1998). Composition of paternal mitochondrial DNA in cloned bovine embryos. FEBS Lett., 426: 352-356.

Sutherland B., S tewart D., Kenchington E.R., Zouros E. (1998). The fate of paternal mitochondrial DNA in developing female mussels, Mytilus edulis: implications for the mechanism of doubly uniparental inheritance of mitochondrial DNA. Genetics, 148: 341-347. 
Śla s k a B. (2010). Structural genomics of the raccoon dog (Nyctereutes procyonoides procyonoides) (in Polish). Rozprawy Naukowe Uniwersytetu Przyrodniczego w Lublinie, 348, Lublin, Poland, pp. 1-108.

Zh a o X., L i N., Gu o W., H u X., Li u Z., Go ng G., Wang A., F eng J., W u C. (2004). Further evidence for paternal inheritance of mitochondrial DNA in the sheep (Ovis aries). Heredity, 93: 399-403.

Received: 5 IX 2018

Accepted: 10 X 2018 